\title{
LMI based stability analysis and robust controller design for discrete linear repetitive processes
}

\author{
K. Galkowski ${ }^{1}$, J. Lam ${ }^{2}$, E. Rogers ${ }^{3, * \dagger}$, S. Xu ${ }_{5}^{4}$, B. Sulikowski ${ }^{1}$, \\ W. Paszke ${ }^{1}$ and D.H. Owens \\ ${ }^{1}$ Institute of Control and Computation Engineering, University of Zielona Gora, Poland \\ ${ }^{2}$ Dept. of Mechanical Engineering, University of Hong Kong \\ ${ }^{3}$ Department of Electronics and Computer Science, University of Southampton, Southampton SO 17 1BK, U.K. \\ ${ }^{4}$ Department of Automation, Nanjing University of Science and Technology, Nanjing 210094, P.R. China \\ ${ }^{5}$ Department of Automatic Control and Systems Engineering, University of Sheffield, Sheffield S1 3JD, U.K.
}

\section{SUMMARY}

Discrete linear repetitive processes are a distinct class of 2D linear systems with applications in areas ranging from long-wall coal cutting through to iterative learning control schemes. The main feature which makes them distinct from other classes of $2 \mathrm{D}$ linear systems is that information propagation in one of the two independent directions only occurs over a finite duration. This, in turn, means that a distinct systems theory must be developed for them. In this paper, the major new development is that an LMI based reformulation of the stability conditions can used to enable the design of a family of control laws which have a well defined physical basis. It is also noted that this setting can be used to investigate robustness aspects. Copyright (C) 2003 John Wiley \& Sons, Ltd.

KEY WORDS: 2D linear systems; LMI; repetitive dynamics; stability; controller design

\section{INTRODUCTION}

The essential unique characteristic of a repetitive, or multipass, process is a series of sweeps, termed passes, through a set of dynamics defined over a fixed finite duration known as the pass length. On each pass an output, termed the pass profile, is produced which acts as a forcing function on, and hence contributes to, the dynamics of the new pass profile. This, in turn, leads to the unique control problem for these processes in that the output sequence of pass profiles generated can contain oscillations that increase in amplitude in the pass to pass direction.

To introduce a formal definition, let $\alpha<+\infty$ denote the pass length (assumed constant). Then in a repetitive process the pass profile $y_{k}(p), 0 \leqslant p \leqslant \alpha$, generated on pass $k$ acts as a forcing function on, and hence contributes to, the dynamics of the new pass profile $y_{k+1}(p), 0 \leqslant p \leqslant \alpha$, $k \geqslant 0$.

\footnotetext{
*Correspondence to: E. Rogers, Department of Electronics and Computer Science, University of Southampton, Highfield, Southampton, SO 17 1BK, U.K.

†E-mail: etar@ecs.soton.ac.uk

Published online 26 June 2003

Copyright (C) 2003 John Wiley \& Sons, Ltd.

Received 11 March 2002

Revised 2 August 2002 Accepted 2 December 2002
} 
Physical examples of repetitive processes include long-wall coal cutting and metal rolling operations (see, for example Reference [1]). Also in recent years applications have arisen where adopting a repetitive process setting for analysis has distinct advantages over alternatives. Examples of these so-called algorithmic applications of repetitive processes include classes of iterative learning control (ILC) schemes [2] and iterative algorithms for solving nonlinear dynamic optimal control problems based on the maximum principle [3]. In the case of iterative learning control for the linear dynamics case, the stability theory for so-called differential and discrete linear repetitive processes is the essential basis for a rigorous stability/convergence theory for such algorithms.

Attempts to control these processes using standard (or 1D) systems theory/algorithms fail (except in a few very restrictive special cases) precisely because such an approach ignores their inherent $2 \mathrm{D}$ systems structure, i.e. information propagation occurs from pass to pass and along a given pass. In seeking a rigorous foundation on which to develop a control theory for these processes, it is natural to attempt to exploit structural links which exist between, in particular, the class of so-called discrete linear repetitive processes and 2D linear systems described by the extensively studied Roesser [4] or Fornasini Marchesini [5] state space models. The discrete linear repetitive processes considered in this contribution are distinct from such 2D linear systems in the sense that information propagation in one of the two separate directions (along the pass) only occurs over a finite duration and hence large key elements of existing 2D systems theory can either (i) not be applied, or (ii) only applied after significant modifications.

A rigorous stability theory for linear repetitive processes has been developed. This theory [6] is based on an abstract model in a Banach space setting which includes all such processes as special cases. Also the results of applying this theory to a wide range of cases has been reported, including the sub-class considered here. This has resulted in stability tests that can be implemented by direct application of well known 1D linear systems tests.

In this paper, we show that an LMI re-formulation of the stability conditions leads naturally to design algorithms for control laws of the type discussed above. Moreover, it is noted that this setting also enables progress to be made on the currently (very much) open problem of stability and control of these processes in the presence of uncertainty in the model structure. Preliminary versions of some of the analysis given here have been given in References [7, 8]. We begin in the next section with a summary of the relevant background results.

\section{BACKGROUND}

Following Rogers and Owens [6] the state-space model of a discrete linear repetitive process has the following form over $0 \leqslant p \leqslant \alpha, k \geqslant 0$

$$
\begin{aligned}
& x_{k+1}(p+1)=A x_{k+1}(p)+B u_{k+1}(p)+B_{0} y_{k}(p) \\
& y_{k+1}(p)=C x_{k+1}(p)+D u_{k+1}(p)+D_{0} y_{k}(p)
\end{aligned}
$$

Here on pass $k, x_{k}(p)$ is the $n \times 1$ state vector, $y_{k}(p)$ is the $m \times 1$ vector pass profile, and $u_{k}(p)$ is the $l \times 1$ vector of control inputs.

To complete the process description, it is necessary to specify the 'initial conditions'-termed the boundary conditions here, i.e. the state initial vector on each pass and the initial pass profile. Here no loss of generality arises from assuming $x_{k+1}(0)=d_{k+1}, k \geqslant 0$, and $y_{0}(p)=f(p)$, where 
$d_{k+1}$ is an $n \times 1$ vector with known constant entries and $f(p)$ is an $m \times 1$ vector whose entries are known functions of $p$.

The abstract model based stability theory [6] for linear repetitive processes consists of two distinct concepts termed asymptotic stability and stability along the pass, respectively. Noting again the unique control problem for these processes, this theory demands that bounded sequences of inputs (the element for each pass is formed from the control inputs, state initial conditions and disturbances which enter on this pass) produce bounded sequences of pass profiles where here 'bounded' is defined in terms of the norm on the underlying function space. The essential difference between them is that asymptotic stability demands this property over the finite pass length whereas stability along the pass is stronger in that it demands this property uniformly, i.e. independent of the pass length.

In the case of processes described by (1), it can be shown [6] that asymptotic stability holds if, and only if, $r\left(D_{0}\right)<1$ where $\mathrm{r}(\cdot)$ denotes the spectral radius. Also if the example under consideration is asymptotically stable and the control input sequence applied $\left\{u_{k}\right\}_{k \geqslant 1}$ converges strongly to $u_{\infty}$ as $k \rightarrow \infty$ then the resulting output pass profile sequence $\left\{y_{k}\right\}_{k \geqslant 1}$ converges strongly to $y_{\infty}$-the so-called limit profile defined (with $D=0$ for ease of presentation) over $0 \leqslant p \leqslant \alpha$ by

$$
\begin{aligned}
& x_{\infty}(p+1)=\left(A+B_{0}\left(I_{m}-D_{0}\right)^{-1} C\right) x_{\infty}(p)+B u_{\infty}(p) \\
& y_{\infty}(p)=\left(I_{m}-D_{0}\right)^{-1} C x_{\infty}(p)
\end{aligned}
$$

In effect, this result states that if a process is asymptotically stable then its repetitive dynamics can, after a 'sufficiently large' number of passes, be replaced by those of a 1D discrete linear system. Note, however, that this property does not guarantee that the limit profile is stable in the normal sense, i.e. $r\left(A+B_{0}\left(I_{m}-D_{0}\right)^{-1} C\right)<1$-a point which is easily illustrated by, for example, the case when $A=-0.5, B=0, B_{0}=0.5+b_{0}, C=1, D=D_{0}=0$, and the real scalar $b_{0}$ is chosen such that $\left|b_{0}\right| \geqslant 1$.

Stability along the pass prevents cases such as the simple example above from arising and in this work it is the following characterization [6] of this property that will be required.

\section{Theorem 1}

Discrete linear repetitive processes described by (1) are stable along the pass if, and only if, the 2D characteristic polynomial

$$
C\left(z_{1}, z_{2}\right):=\operatorname{det}\left[\begin{array}{cc}
I_{n}-z_{1} A & -z_{1} B_{0} \\
-z_{2} C & I_{m}-z_{2} D_{0}
\end{array}\right] \neq 0 \quad \forall\left(z_{1}, z_{2}\right) \in \bar{U}^{2}
$$

where $\bar{U}^{2}=\left\{\left(z_{1}, z_{2}\right):\left|z_{1}\right| \leqslant 1,\left|z_{2}\right| \leqslant 1\right\}$.

Note that stability along the pass includes asymptotic stability as a special case ((3) clearly requires that $\left.r\left(D_{0}\right)<1\right)$.

Note that (3) is also the necessary and sufficient condition for stability of a 2D discrete linear system described by a Roesser model whose state dynamics are defined by the state space matrices $\left\{A, B, B_{0}, C, D_{0}\right\}$. This is a well known fact [6] and leads immediately to the interchange of stability tests for these two classes of linear systems. None of these tests, however, provide an effective basis on which to design control laws for these processes beyond the task of obtaining conditions for stability of the resulting closed loop system. In the case of repetitive processes 
and, in particular, applications areas (such as ILC) it is also essential to include performance in the design specification.

The remainder of this paper shows that an LMI formulation meets this last specification for a physically motivated control law but at the expense of a sufficient but not necessary condition for stability along the pass. Moreover, it is shown that the LMI setting leads to a Lyapunov equation which can be used to extract computable information on a key performance measure, i.e. the rate of approach of the output sequence of pass profiles to the resulting limit profile for a stable along the pass example. Finally, it is noted that the LMI setting also allows a start to be made on robustness analysis for these processes both open loop and closed loop under control action.

\section{LMI BASED STABILITY ANALYSIS}

In this work, the following well known (for a proof see Reference [9]) result is extensively used.

\section{Lemma 1}

Given constant matrices $W, L$, and $V$ of appropriate dimensions where $W=W^{\mathrm{T}}$ and $V=V^{\mathrm{T}}>$ 0 , then

$$
W+L^{\mathrm{T}} V L<0
$$

if and only if

$$
\left[\begin{array}{cc}
W & L^{\mathrm{T}} \\
L & -V^{-1}
\end{array}\right]<0
$$

or, equivalently,

$$
\left[\begin{array}{cc}
-V^{-1} & L \\
L^{\mathrm{T}} & W
\end{array}\right]<0
$$

The matrix $W+L^{\mathrm{T}} V L$ is known as the Schur complement of the matrix in (5) or (6).

Now define the following matrices from the state space model (1):

$$
\hat{A}_{1}=\left[\begin{array}{cc}
A & B_{0} \\
0 & 0
\end{array}\right], \quad \hat{A}_{2}=\left[\begin{array}{cc}
0 & 0 \\
C & D_{0}
\end{array}\right]
$$

Then we have the following sufficient condition for stability along the pass of processes described by (1) (with the assumed boundary conditions).

Theorem 2

Discrete linear repetitive processes described by (1) are stable along the pass if $\exists$ matrices $P=P^{\mathrm{T}}>0$ and $Q=Q^{\mathrm{T}}>0$ satisfying the following LMI:

$$
\left[\begin{array}{cc}
\hat{A}_{1}^{\mathrm{T}} P \hat{A}_{1}+Q-P & \hat{A}_{1}^{\mathrm{T}} P \hat{A}_{2} \\
\hat{A}_{2}^{\mathrm{T}} P \hat{A}_{1} & \hat{A}_{2}^{\mathrm{T}} P \hat{A}_{2}-Q
\end{array}\right]<0
$$


Proof

Using * to denote the complex conjugate transpose operation, first pre-multiply (8) by $\left[z_{1}^{*} I_{n}\right.$ $\left.z_{2}^{*} I_{m}\right]$ and post-multiply it by the complex conjugate transpose of this last matrix to yield

$$
\left(z_{1} \hat{A}_{1}+z_{2} \hat{A}_{2}\right)^{*} P\left(z_{1} \hat{A}_{1}+z_{2} \hat{A}_{2}\right)+z_{1}^{*} Q z_{1}-z_{1}^{*} P z_{1}-z_{2}^{*} Q z_{2}<0
$$

Also for (8) to hold we must have that $Q-P<0$ and hence $\left(\forall\left(z_{1}, z_{2}\right) \in \bar{U}^{2}\right)$

$$
\begin{aligned}
z_{1}^{*} Q z_{1}-z_{1}^{*} P z_{1}-z_{2}^{*} Q z_{2} & =z_{1}^{*}(Q-P) z_{1}-z_{2}^{*} Q z_{2} \\
& =-\left[z_{1}^{*}(P-Q) z_{1}+z_{2}^{*} Q z_{2}\right] \\
& \geqslant-[P-Q+Q]=-P
\end{aligned}
$$

Consequently for (8) to hold $r\left(z_{1} \hat{A}_{1}+z_{2} \hat{A}_{2}\right)<1, \forall\left(z_{1}, z_{2}\right) \in \bar{U}^{2}$. This in turn implies that

$$
\operatorname{det}\left(I_{n+m}-z_{1} \hat{A}_{1}-z_{2} \hat{A}_{2}\right) \neq 0, \forall\left(z_{1}, z_{2}\right) \in \bar{U}^{2}
$$

which is equivalent to (3) and the proof is complete.

The following corollary to Theorem 2 links the LMI result derived here to a Lyapunov equation interpretation of stability along the pass and will be used in the next section to obtain computable information on the rate of convergence of the output sequence of pass profiles $\left\{y_{k}\right\}_{k \geqslant 1}$ produced by a stable along the pass process.

\section{Corollary 1}

Discrete linear repetitive processes described by (1) are stable along the pass if $\exists$ matrices $W_{1}=W_{1}^{\mathrm{T}}>0$ and $W_{2}=W_{2}^{\mathrm{T}}>0$ such that the Lyapunov inequality

$$
\Phi^{\mathrm{T}} W \Phi-W<0
$$

holds, where $\Phi$ is the so-called augmented plant matrix defined by

$$
\Phi=\left[\begin{array}{ll}
A & B_{0} \\
C & D_{0}
\end{array}\right]=\hat{A}_{1}+\hat{A}_{2}
$$

and $W=W_{1} \oplus W_{2}$ where $\oplus$ denotes the direct sum, i.e. $W=\operatorname{diag}\left\{W_{1}, W_{2}\right\}$.

Proof

If (9) holds, then $\exists$ a sufficiently small scalar $\varepsilon>0$ such that

$$
\Phi^{\mathrm{T}} W \Phi-W+\varepsilon I<0
$$

or, on substituting for $\Phi$ in terms of $\hat{A}_{1}$ and $\hat{A}_{2}$,

$$
\hat{A}_{1}^{\mathrm{T}} W \hat{A}_{1}+\hat{A}_{2}^{\mathrm{T}} W \hat{A}_{2}-W+\varepsilon I<0
$$


Note also that $\hat{A}_{1}^{\mathrm{T}} W \hat{A}_{2}=0$, and define $Q$ and $P$ of Theorem 2 as $Q=\hat{A}_{2}^{\mathrm{T}} W \hat{A}_{2}+\varepsilon I$, and $P=W$ respectively. Then (12) can be rewritten in the analogous form to (8) as

$$
\left[\begin{array}{cc}
\hat{A}_{1}^{\mathrm{T}} P \hat{A}_{1} & \hat{A}_{1}^{\mathrm{T}} P \hat{A}_{2} \\
\hat{A}_{2}^{\mathrm{T}} P \hat{A}_{1} & \hat{A}_{2}^{\mathrm{T}} P \hat{A}_{2}-Q
\end{array}\right]<0
$$

and the proof is complete.

\section{LMI BASED CONTROLLER DESIGN}

The design of control laws for 2D discrete linear systems described by the Roesser [4] and Fornasini Marchesini [5] state space models has received considerable attention in the literature over the years. A valid criticism of such work, however, is that the structure and design of the control algorithms are not well founded physically due to the fact that, for example, the concept of a state for these systems is not uniquely defined. For example, it is possible to define a state feedback law based on the local or global state vectors. Also in the absence of generalizations of well defined and understood 1D concepts, e.g. the pole assignment problem and error actuated output feedback control action, it has not been really possible to formulate a control design problem beyond that of obtaining conditions for stabilization under the control action.

This difficulty does not arise with linear repetitive processes. For example, it is physically meaningful to define the current pass error as the difference, at each point along the pass, between a specified reference trajectory for that pass (which in most cases will be the same on each pass) and the actual pass profile produced. Then one can define a so-called current pass error actuated controller which uses the generated error vector to construct the current trial control input vector. In which context, preliminary work (see, for example Reference [10]) has shown that, except in a few very restrictive special cases, the controller used must be actuated by a combination of current trial information and 'feedforward' information from the previous pass to guarantee even stability along the pass closed loop. (Note that in the ILC application area the previous pass output vector is an obvious signal to use as feedforward action.)

As a major systematic attempt at controller design for discrete linear repetitive processes we consider a control law of the following form over $0 \leqslant p \leqslant \alpha, k \geqslant 0$

$$
u_{k+1}(p)=K_{1} x_{k+1}(p)+K_{2} y_{k}(p):=K\left[\begin{array}{c}
x_{k+1}(p) \\
y_{k}(p)
\end{array}\right]
$$

where $K_{1}$ and $K_{2}$ are appropriately dimensioned matrices to be designed. In effect, this control law uses feedback of the current pass state vector (which is assumed to be available for use) and 'feedforward' of the previous pass profile vector. (Note that in repetitive process theory the term feedforward is used to describe the case where (state or pass profile) information from the previous pass (or passes) is used as (part of) the input to a control law applied on the current pass, i.e. to information which is propagated in the pass to pass $(k)$ direction.)

This control law has clear physical meaning for practical applications of discrete linear repetitive processes and the obvious questions now to ask are: (i) can we obtain conditions for closed loop stability along the pass coupled with easily (in relative terms) applied algorithms for computing the controller parameters? and (ii) can we also obtain computable information as to expected closed loop performance which is relevant to what may be demanded of such a closed 
loop process? Here we show that the answer to both these questions is yes if the LMI setting of the previous section is used. (Note that there may well be alternative approaches to these questions - if this is indeed the case then the results here will serve as a baseline comparison.)

Application of the control law (14) to (1) results in the following condition for closed loop stability along the pass on applying the necessary and sufficient conditions of Theorem 1 to the resulting closed loop state space model

$$
C_{c}\left(z_{1}, z_{2}\right) \neq 0, \forall\left(z_{1}, z_{2}\right) \in \bar{U}^{2}
$$

where

$$
C_{c}\left(z_{1}, z_{2}\right):=\operatorname{det}\left[I-\left[\begin{array}{ll}
z_{1}\left(A+B K_{1}\right) & z_{1}\left(B_{0}+B K_{2}\right) \\
z_{2}\left(C+D K_{1}\right) & z_{2}\left(D_{0}+D K_{2}\right)
\end{array}\right]\right]
$$

Now introduce the matrices

$$
\hat{B}_{1}=\left[\begin{array}{l}
B \\
0
\end{array}\right], \quad \hat{B}_{2}=\left[\begin{array}{l}
0 \\
D
\end{array}\right]
$$

Then we have the following result which is proved by simply repeating the proof of Theorem 2 for the closed loop system which results from application of the control law (14) and hence the details are omitted.

\section{Theorem 3}

Suppose that a discrete linear repetitive process of the form described by (1) is subjected to a control law of the form (14). Then the resulting closed loop process is stable along the pass if $\exists$ matrices $P=P^{\mathrm{T}}>0$ and $Q=Q^{\mathrm{T}}>0$ such that

$$
\left[\begin{array}{rc}
\left(\hat{A}_{1}^{\mathrm{T}}+K^{\mathrm{T}} \hat{B}_{1}^{\mathrm{T}}\right) P\left(\hat{A}_{1}+\hat{B}_{1} K\right)+Q-P & \left(\hat{A}_{1}^{\mathrm{T}}+K^{\mathrm{T}} \hat{B}_{1}^{\mathrm{T}}\right) P\left(\hat{A}_{2}+\hat{B}_{2} K\right) \\
\left(\hat{A}_{2}^{\mathrm{T}}+K^{\mathrm{T}} \hat{\boldsymbol{B}}_{2}^{\mathrm{T}}\right) P\left(\hat{A}_{1}+\hat{B}_{1} K\right) & \left(\hat{A}_{2}^{\mathrm{T}}+K^{\mathrm{T}} \hat{\boldsymbol{B}}_{2}^{\mathrm{T}}\right) P\left(\hat{A}_{2}+\hat{B}_{2} K\right)-Q
\end{array}\right]<0
$$

The major remaining difficulty with the matrix inequality of Theorem 3 is that it is nonlinear in its parameters. It can, however, be converted into the following result where the inequality is a strict LMI with a linear constraint which also gives a formula for computing $K$ in (14).

\section{Theorem 4}

The condition of Theorem 3 is equivalent to the requirement that $\exists$ matrices $Y=Y^{\mathrm{T}}>0$, $Z=Z^{\mathrm{T}}>0$, and $N$ such that the following LMI holds.

$$
\left[\begin{array}{ccc}
Z-Y & 0 & Y \hat{A}_{1}^{\mathrm{T}}+N^{\mathrm{T}} \hat{B}_{1}^{\mathrm{T}} \\
0 & -Z & Y \hat{A}_{2}^{\mathrm{T}}+N^{\mathrm{T}} \hat{B}_{2}^{\mathrm{T}} \\
\hat{A}_{1} Y+\hat{B}_{1} N & \hat{A}_{2} Y+\hat{B}_{2} N & -Y
\end{array}\right]<0
$$

If (19) holds, then a stabilizing $K$ in the control law (14) is given by (see also Reference [11] for the origins of this formula (and (26) below) in standard systems theory)

$$
K=N Y^{-1}
$$


Proof

First apply the Schur's complement formula of (5) to (18), with

$$
W=\left[\begin{array}{cc}
Q-P & 0 \\
0 & -Q
\end{array}\right], \quad V=P^{-1}, \quad L=P\left[\left(\hat{A}_{1}+\hat{B}_{1} K\right)\left(\hat{A}_{2}+\hat{B}_{2} K\right)\right] .
$$

Now left and right multiply the result from this step by $\operatorname{diag}\left(P^{-1}, P^{-1}, P^{-1}\right)$, and then make the following substitutions:

$$
Z=P^{-1} Q P^{-1}>0, \quad Y=P^{-1}>0
$$

to yield

$$
\left[\begin{array}{ccc}
Z-Y & 0 & Y\left(\hat{A}_{1}^{\mathrm{T}}+K^{\mathrm{T}} \hat{B}_{1}^{\mathrm{T}}\right) \\
0 & -Z & Y\left(\hat{A}_{2}^{\mathrm{T}}+K^{\mathrm{T}} \hat{B}_{2}^{\mathrm{T}}\right) \\
\left(\hat{A}_{1}+\hat{B}_{1} K\right) Y & \left(\hat{A}_{2}+\hat{B}_{2} K\right) Y & -Y
\end{array}\right]<0
$$

Application of the formula given by (20) now completes the proof.

An alternative design is possible via the 2D Lyapunov inequality (9). In particular, write

$$
R=\left[\begin{array}{ll}
B & 0 \\
0 & D
\end{array}\right], \quad K=\left[\begin{array}{ll}
K_{1} & K_{2} \\
K_{1} & K_{2}
\end{array}\right]
$$

Then interpreting (9) closed loop gives the following sufficient condition for stability along the pass

$$
(\Phi+R K)^{\mathrm{T}} W(\Phi+R K)-W<0
$$

where (as before) $W=W_{1} \oplus W_{2}$, and the following result can now be established.

\section{Theorem 5}

Suppose that a discrete linear repetitive process of the form described by (1) is subjected to a control law of the form (14). Then the resulting closed loop process is stable along the pass if $\exists$ matrices $P=P_{1} \oplus P_{2}>0, P_{1}=P_{1}^{\mathrm{T}}>0, P_{2}=P_{2}^{\mathrm{T}}>0$ and $N=\left[\begin{array}{ll}N_{1} & N_{2} \\ N_{1} & N_{2}\end{array}\right]$ such that

$$
\left[\begin{array}{cc}
-P & \Phi P+R N \\
P \Phi^{\mathrm{T}}+N^{\mathrm{T}} R^{\mathrm{T}} & -P
\end{array}\right]<0
$$

holds. Also a stabilizing $K$ in the control law (14) is given by

$$
K=N P^{-1}
$$

Proof

First apply the Schur's complement formula (6) to (24) to obtain

$$
\left[\begin{array}{cc}
-W^{-1} & \Phi+R K \\
(\Phi+R K)^{\mathrm{T}} & -W
\end{array}\right]<0
$$


Multiplying (27) from the left and right by $\operatorname{diag}\{W, I\}$ now yields

$$
\left[\begin{array}{cc}
-W & W(\Phi+R K) \\
(\Phi+R K)^{\mathrm{T}} W & -W
\end{array}\right]<0
$$

Now introduce the substitution $P=W^{-1}$, and then multiply the result from the left and right by $\operatorname{diag}\{P, P\}$ to yield

$$
\left[\begin{array}{cc}
-P & \Phi P+R K P \\
P \Phi^{\mathrm{T}}+P K^{\mathrm{T}} R^{\mathrm{T}} & -P
\end{array}\right]<0
$$

and the proof is completed by substitution from (26).

One important point to note about the results to date in this section is that the LMI decision variables $Y$ and $P$ in Theorems 4 and 5, respectively are subsequently directly involved in the computation of the resulting controller (via (20) and (26) respectively). This could be a source of further conservatism and a similar problem has been encountered in 1D discrete linear systems theory. This has been considered in References $[12,13]$ and here we extend these ideas to the case of discrete linear repetitive processes, starting with the following result as an alternative to Theorem 5.

\section{Theorem 6}

Suppose that a discrete linear repetitive process of the form described by (1) is subjected to a control law of the form (14). Then the resulting closed loop process is stable along the pass if $\exists$ matrices $W_{1}=W_{1}^{\mathrm{T}}>0, W_{2}=W_{2}^{\mathrm{T}}>0, Q=Q^{\mathrm{T}}>0$ and $G_{1}, G_{2}, N_{1}$, and $N_{2}$ such that

$$
\left[\begin{array}{cc}
-W & \Phi G+R N \\
(\Phi G+R N)^{\mathrm{T}} & -G-G^{\mathrm{T}}+W
\end{array}\right]<0
$$

holds, where $W=W_{1} \oplus W_{2}, G=G_{1} \oplus G_{2}$, (and $R$ is again defined as in (23)). Also a stabilizing $K$ in the control law (14) is given by

$$
K=N G^{-1}
$$

\section{Proof}

First note that $G$ is invertible since from (30) $G+G^{\mathrm{T}}>0$. (Note also that the special case when $G=P$ recovers the result of Theorem 5.) Now left multiply (30) by $\left[I R N G^{-1}\right]$ and right multiply by the transpose of this last matrix. Then on substituting from (31) we obtain the Lyapunov inequality (24) and the proof is complete.

In actual fact, it is possible to weaken the block diagonal matrix assumptions required in this last result and hence reduce a possible source of conservativeness in the resulting stability along the pass condition. In particular, we have the following result.

\section{Theorem 7}

Suppose that a discrete linear repetitive process of the form described by (1) is subjected to a control law of the form (14). Then the resulting closed loop process is stable along the pass 
if $\exists$ matrices $P=P^{\mathrm{T}}>0, Q=Q^{\mathrm{T}}>0, G=G_{1} \oplus G_{2}$, and $N=N_{1} \oplus N_{2}$ such that

$$
\left[\begin{array}{ccc}
Q-P & 0 & \tilde{A}_{1} \hat{G}_{1}+R \hat{N}_{1} \\
0 & -Q & \tilde{A}_{2} \hat{G}_{2}+R \hat{N}_{2} \\
\left(\tilde{A}_{1} \hat{G}_{1}+R \hat{N}_{1}\right)^{\mathrm{T}} & \left(\tilde{A}_{2} \hat{G}_{2}+R \hat{N}_{2}\right)^{\mathrm{T}} & P-G-G^{\mathrm{T}}
\end{array}\right]<0
$$

where

$$
\begin{aligned}
& \tilde{A}_{1}=\left[\begin{array}{ll}
A & 0 \\
C & 0
\end{array}\right], \quad \tilde{A}_{2}=\left[\begin{array}{ll}
0 & B_{0} \\
0 & D_{0}
\end{array}\right] \\
& \hat{N}_{1}=\left[\begin{array}{ll}
N_{1} & 0 \\
N_{1} & 0
\end{array}\right], \quad \hat{N}_{2}=\left[\begin{array}{ll}
0 & N_{2} \\
0 & N_{2}
\end{array}\right] \\
& \hat{G}_{1}=\left[\begin{array}{cc}
G_{1} & 0 \\
0 & 0
\end{array}\right], \quad \hat{G}_{2}=\left[\begin{array}{ll}
0 & 0 \\
0 & G_{2}
\end{array}\right]
\end{aligned}
$$

and $R$ is defined in (23).

Proof
This follows immediately on left multiplying (32) by $\left[I \mid \begin{array}{l}Y_{1} \\ Y_{2}\end{array}\right]$ and right multiplying this same equation by the transpose of this matrix to obtain

$$
\left[\begin{array}{cc}
Y_{1} P Y_{1}^{\mathrm{T}}+Q-P & Y_{1} P Y_{2}^{\mathrm{T}} \\
Y_{2} P Y_{1}^{\mathrm{T}} & Y_{2} P Y_{2}^{\mathrm{T}}-Q
\end{array}\right]<0
$$

where

$$
\begin{aligned}
& Y_{1}=\tilde{A}_{1}+\left[\begin{array}{ll}
B N_{1} G_{1}^{-1} & 0 \\
D N_{1} G_{1}^{-1} & 0
\end{array}\right] \\
& Y_{2}=\tilde{A}_{2}+\left[\begin{array}{ll}
0 & B N_{2} G_{2}^{-1} \\
0 & D N_{2} G_{2}^{-1}
\end{array}\right]
\end{aligned}
$$

i.e. the 2D Lyapunov equation based sufficient condition for closed loop stability along the pass and the proof is complete.

Now we give the following example of the application of Theorem 4 as an illustration of the new results developed in this paper. This is the special case of (1) defined by

$$
A=\left[\begin{array}{ccc}
-1.1 & 0.2 & 0.1 \\
0.1 & -1.2 & 0.2 \\
-2 & -0.3 & -1.2
\end{array}\right], \quad B_{0}=\left[\begin{array}{cccc}
-0.1 & -0.5 & -0.4 & 0.1 \\
-0.7 & 1.2 & -0.5 & 0.5 \\
-0.5 & 0.4 & -0.5 & -0.5
\end{array}\right]
$$




$$
\begin{gathered}
C=\left[\begin{array}{ccc}
-1.05 & -0.05 & 0.2 \\
-0.3 & -0.2 & -0.2 \\
0.2 & -2.1 & 0.2 \\
-0.1 & 0.1 & -0.1
\end{array}\right], \quad D_{0}=\left[\begin{array}{cccc}
-0.1 & 0.2 & 0.2 & 0.1 \\
0.4 & 0.2 & 0.1 & 0.2 \\
0.2 & 0.2 & -0.1 & 0.1 \\
0.2 & 0 & -0.2 & -0.1
\end{array}\right] \\
B=\left[\begin{array}{cc}
-0.1 & 0.2 \\
0.3 & 0.2 \\
0.2 & 0.2
\end{array}\right], \quad D=\left[\begin{array}{cc}
-0.1 & 0.5 \\
0.7 & 0.2 \\
-0.5 & 0.4 \\
-0.2 & -0.1
\end{array}\right]
\end{gathered}
$$

This process is unstable along the pass since (by direct computation) the matrix $A$ has at least one eigenvalue with modulus greater than unity and hence (3) does not hold. Use of a routine from the MATLAB LMI toobox now shows that (19) of Theorem 4 is satisfied in this case by

$$
\begin{aligned}
N & =\left[\begin{array}{ccccccc}
-2.8115 & -1.4622 & 16.0718 & -18.1474 & -12.8291 & 13.8705 & 0.6210 \\
39.0056 & 32.7175 & -35.7558 & -17.9019 & -9.3048 & 0.8362 & -5.1715
\end{array}\right] \\
Z & =\left[\begin{array}{ccccccc}
5.1525 & 2.6612 & -4.7635 & -2.4572 & -2.1636 & -2.4726 & -0.9774 \\
2.6612 & 5.8978 & -1.6409 & -1.0039 & 1.9362 & 0.8944 & 0.7474 \\
-4.7635 & -1.6409 & 11.1693 & 0.6381 & 0.7004 & 1.7970 & -3.3638 \\
-2.4572 & -1.0039 & 0.6381 & 13.2382 & 8.9026 & -11.2128 & -3.0147 \\
-2.1636 & 1.9362 & 0.7004 & 8.9026 & 8.7124 & -4.9491 & -2.7050 \\
-2.4726 & 0.8944 & 1.7970 & -11.2128 & -4.9491 & 17.4904 & 5.7660 \\
-0.9774 & 0.7474 & -3.3638 & -3.0147 & -2.7050 & 5.7660 & 13.0843
\end{array}\right] \\
Y & =\left[\begin{array}{cccccccc}
13.3828 & 7.5835 & -15.1242 & -2.2763 & -2.7902 & -5.0771 & -3.1627 \\
7.5835 & 10.7170 & -7.2051 & 1.5330 & 3.8629 & -1.9397 & -0.4330 \\
-15.1242 & -7.2051 & 35.0306 & 0.0373 & -1.5865 & 0.6176 & -2.3396 \\
-2.2763 & 1.5330 & 0.0373 & 20.1395 & 14.9850 & -15.0870 & -6.3348 \\
-2.7902 & 3.8629 & -1.5865 & 14.9850 & 16.2573 & -7.2847 & -7.9448 \\
-5.0771 & -1.9397 & 0.6176 & -15.0870 & -7.2847 & 25.7798 & 9.2018 \\
-3.1627 & -0.4330 & -2.3396 & -6.3348 & -7.9448 & 9.2018 & 28.4592
\end{array}\right]
\end{aligned}
$$

and also (where $(\cdot)$ denotes the set of eigenvalues of the matrix argument) 


$$
\operatorname{eig}(Y)=\left[\begin{array}{c}
1.3876 \\
0.0142 \\
9.8029 \\
16.2720 \\
22.5253 \\
45.5108 \\
54.2533
\end{array}\right], \quad \operatorname{eig}(Z)=\left[\begin{array}{c}
0.2139 \\
0.0084 \\
4.3234 \\
8.0831 \\
12.3740 \\
16.4477 \\
33.2945
\end{array}\right]
$$

Hence a controller of the form (14) which guarantees closed loop stability along the pass is defined by the following matrices

$$
\begin{gathered}
K_{1}=\left[\begin{array}{ccc}
-1.5902 & 1.4529 & -0.0192 \\
8.2022 & -2.2933 & 2.3288
\end{array}\right] \\
K_{2}=\left[\begin{array}{cccc}
-0.5497 & -1.2365 & -0.1375 & -0.5575 \\
-1.9194 & 4.5352 & 1.0874 & 1.3736
\end{array}\right]
\end{gathered}
$$

The corresponding matrices to $A, B_{0}, C$, and $D_{0}$ in the closed loop stable along the pass process are then given by

$$
\begin{aligned}
A_{\text {new }}= & {\left[\begin{array}{ccc}
0.6995 & -0.4039 & 0.5677 \\
1.2634 & -1.2228 & 0.6600 \\
-0.6776 & -0.4681 & -0.7381
\end{array}\right], } \\
B_{\text {nnew }}= & {\left[\begin{array}{cccc}
-0.4289 & 0.5307 & -0.1688 & 0.4305 \\
-1.2488 & 1.7361 & -0.3238 & 0.6075 \\
-0.9938 & 1.0597 & -0.3100 & -0.3368
\end{array}\right] } \\
C_{\text {new }}= & {\left[\begin{array}{cccc}
3.2101 & -1.3419 & 1.3663 \\
0.2273 & 0.3583 & 0.2524 \\
4.2760 & -3.7438 & 1.1411 \\
-0.6022 & 0.0388 & -0.3291
\end{array}\right], } \\
D_{0 \text { new }}= & {\left[\begin{array}{cccc}
-1.0047 & 2.5913 & 0.7575 & 0.8425 \\
-0.3687 & 0.2415 & 0.2212 & 0.0845 \\
-0.2929 & 2.6323 & 0.4037 & 0.9282 \\
0.5019 & -0.2062 & -0.2812 & -0.1259
\end{array}\right] }
\end{aligned}
$$

The existence of examples for which Theorem 4 holds when Theorem 5 only produces an inconclusive result is perhaps not surprising given in the former the LMI analysis yields an 
unstructured $P$ but use of Theorem 5 requires a structured solution $W=W_{1} \oplus W_{2}$ of (9) closed loop.

If a discrete linear repetitive process is stable along the pass then the sequence of pass profiles generated $\left\{y_{k}\right\}_{k \geqslant 1}$ converges at a geometric rate (which is independent of the pass length) to the resulting limit profile which is guaranteed to be a stable 1D discrete linear system. Hence any form of computational information concerning this convergence rate would clearly be of interest open or closed loop. In what follows, we show that if (9) holds open loop or Theorem 5 closed loop, then it is possible to use the solution matrix $W$ to extract computable information on the rate of convergence to the resulting limit profile.

Suppose, therefore, that (9) holds for the example under consideration and that this example is operating under a zero current pass input sequence and introduce

$$
\begin{aligned}
& \left\|x_{k+1}(p+1)\right\|_{W_{1}}^{2}:=x_{k+1}^{\mathrm{T}}(p+1) W_{1} x_{k+1}(p+1) \\
& \left\|y_{k+1}(p)\right\|_{W_{2}}^{2}:=y_{k+1}^{\mathrm{T}}(p) W_{2} y_{k+1}(p)
\end{aligned}
$$

Note also that (9) is equivalent to $\exists W=W_{1} \oplus W_{2}=W^{\mathrm{T}}>0$ such that

$$
\Phi^{\mathrm{T}} W \Phi-W=-I
$$

Then on pre-multiplying this last equation by $\left[x_{k}^{\mathrm{T}}(p), y_{k}^{\mathrm{T}}(p)\right]$ and post-multiplying by the transpose of this vector yields in terms of (36)

$$
\left\|x_{k+1}(p+1)\right\|_{W_{1}}^{2}+\left\|y_{k+1}(p)\right\|_{W_{2}}^{2}-\left\|x_{k}(p)\right\|_{W_{1}}^{2}-\left\|y_{k}(p)\right\|_{W_{2}}^{2}=-\left\|x_{k}(p)\right\|_{I}^{2}-\left\|y_{k}(p)\right\|_{I}^{2}
$$

Also no loss of generality arises from assuming $x_{k+1}(0)=0, k \geqslant 0$, and introduce

$$
\begin{aligned}
\left\|x_{k}\right\|_{W_{1}}^{2} & :=\sum_{p=0}^{\infty}\left\|x_{k}(p)\right\|_{W_{1}}^{2} \\
\left\|y_{k}\right\|_{W_{2}}^{2} & :=\sum_{p=0}^{\infty}\left\|y_{k}(p)\right\|_{W_{2}}^{2}
\end{aligned}
$$

and writing (38) in terms of these last two quantities gives

$$
\begin{aligned}
\left\|x_{k+1}\right\|_{W_{1}}^{2}+\left\|y_{k+1}\right\|_{W_{2}}^{2} & =\left\|x_{k}\right\|_{W_{1}-I}^{2}+\left\|y_{k}\right\|_{W_{2}-I}^{2} \\
& \leqslant \lambda\left(\left\|x_{k}\right\|_{W_{1}}^{2}+\left\|y_{k}\right\|_{W_{2}}^{2}\right)
\end{aligned}
$$

where (see below) $\lambda$ is a positive constant.

Now assume that

$$
\begin{aligned}
& W_{1}-I \leqslant \lambda_{1} W_{1} \\
& W_{2}-I \leqslant \lambda_{2} W_{2}
\end{aligned}
$$

where $\lambda_{1}$ and $\lambda_{2}$ are positive real scalars. Then

$$
\left\|\left(x_{k+1}, y_{k+1}\right)\right\|^{2}:=\left\|x_{k+1}\right\|_{W_{1}}^{2}+\left\|y_{k+1}\right\|_{W_{2}}^{2} \leqslant \lambda\left(\left\|\left(x_{k}, y_{k}\right)\right\|^{2}\right)
$$

where $\lambda=\max \left(\lambda_{1}, \lambda_{2}\right)$. The process is stable along the pass if $\lambda<1$ which is always guaranteed to be true if $W_{1}, W_{2}>I$. Hence we have geometric convergence to zero in the pass to pass direction of the output sequence of pass profiles. 
It is also possible to compute the rate $\lambda$ using the fact that if a square matrix, say $\hat{W}$, satisfies $\hat{W}=\hat{W}^{\mathrm{T}} \geqslant I$ then $\exists \hat{\lambda} \in[0,1): \hat{W}-I \leqslant \hat{\lambda} \hat{W}$. Then it is easily shown that

$$
\hat{W}-I \leqslant \hat{W}-\frac{1}{\bar{\sigma}^{2}(\hat{W})} \hat{W}=\left(1-\frac{1}{\bar{\sigma}^{2}(\hat{W})}\right) \hat{W}
$$

where $\bar{\sigma}(\cdot)$ denotes the maximum singular value of its matrix argument. Hence in the case of (42), we have that

$$
\lambda=\max \left(1-\frac{1}{\bar{\sigma}^{2}\left(W_{i}\right)}\right), \quad i=1,2
$$

In effect, this result states that if the example under consideration satisfies (9) open loop or Theorem 5 closed loop, then the pass profile sequence generated (and also the current pass state vector sequence) converges to the resulting limit profile at a geometric rate for which a computable upper bound is available from the 2D Lyapunov equation solution matrix. Further research is clearly required to determine exactly how this information can be exploited to best advantage in controller design.

At this stage, it is appropriate if note that (9) with $<0$ replaced by $=-I$ is precisely the Lyapunov equation first obtained in Reference [14] for the Roesser model of a 2D discrete linear system whose dynamics are defined by the state space matrices $\left\{A, B, B_{0}, C, D, D_{0}\right\}$. It is, in general, a sufficient but not necessary condition for stability (either for a $2 \mathrm{D}$ system or stability along the pass of discrete linear repetitive process). This has an identical structure to that for 1D discrete linear systems but is termed '2D' in the literature to highlight the fact that there are essentially two different approaches to developing Lyapunov equations for stability in the setting of $2 \mathrm{D}$ linear systems/repetitive processes. The alternative is the so-called 1D Lyapunov equation where the constant coefficients of the 2D equation are replaced by ones which are functions in a complex variable. (For a detailed treatment of the 1D Lyapunov equation for discrete linear repetitive processes see Reference [15]). The major unique feature of the 2D Lyapunov equation for discrete linear repetitive processes developed here is the performance bounds for a stable along the pass example.

\section{CONCLUSIONS}

This paper has developed an LMI based approach to stability along the pass of discrete linear repetitive processes - a distinct class of $2 \mathrm{D}$ linear systems of both theoretical and applications interest. The major result is that this LMI formulation of stability along the pass can be immediately used to design a powerful class of control laws for these processes which, crucially, have a well defined physical interpretation for applications areas such as iterative learning control. These features are missing from alternative stability characterizations-including those arising from the use of theory first developed for the Roesser model for 2D linear systems (where also control laws are much less related to the underlying dynamics).

The LMI based characterization has also been linked to the so-called 2D Lyapunov equation interpretation of stability along the pass and this leads to computable information on expected performance of examples which are stable along the pass (either open loop or closed loop under control action). In particular, a computable bound on the rate of approach of the output sequence of pass profiles to the resulting limit profile of stable along the pass examples have 
been developed. Such information is again critical to applications - in iterative learning control there is clearly a trade-off between the rate at which the process converges to the learned signal and the dynamics produced along any pass (or trial in iterative learning control terminology).

It is important to place the results of this paper in context-essentially they represent a systematic procedure for stability analysis and onward controller design, as opposed to just stability analysis only, for a very important and distinct class of 2D linear systems using control laws which are well grounded in terms of the underlying process dynamics. One key area for which little or no results are currently available is the stability and control of discrete linear repetitive processes in the presence of uncertainty in the model structure. Here it is possible to immediately see a role for the LMI analysis of this paper.

To detail this last point a little further, define the so-called augmented input matrix for processes described by (1) as

$$
\Psi=\left[\begin{array}{l}
B \\
D
\end{array}\right]
$$

Suppose also that $\Psi$ and the augmented plant matrix $\Phi$ are subject to additive perturbations defined as follows

$$
\begin{aligned}
& \Phi_{p}:=\Phi+\Delta \Phi \\
& \Psi_{p}:=\Psi+\Delta \Psi
\end{aligned}
$$

where

$$
\Delta \Phi=\left[\begin{array}{cc}
\Delta A & \Delta B_{0} \\
\Delta C & \Delta D_{0}
\end{array}\right], \quad \Delta \Psi=\left[\begin{array}{c}
\Delta B \\
\Delta D
\end{array}\right]
$$

Also assume that the uncertainties here have the following typical structure

$$
\left[\begin{array}{ll}
\Delta \Phi & \Delta \Psi
\end{array}\right]=\left[\begin{array}{l}
H_{1} \\
H_{2}
\end{array}\right] F\left[\begin{array}{ll}
E_{1} & E_{2}
\end{array}\right]
$$

where the matrices on the right-hand side are of compatible dimensions and also $F^{\mathrm{T}} F \leqslant I$. It is important to note here that the matrices $H_{i}$ and $E_{i}, i=1,2$, are fixed for a given example but $F$ can be any matrix which satisfies $F^{\mathrm{T}} F \leqslant I$.

Given this setup, it is a routine exercise to show that the analysis of this paper generalizes in a natural manner to processes with this structure and hence the details are omitted here. One counter argument may be that the uncertainty structure used here is well known in the $1 \mathrm{D}$ linear systems area. This is, in fact, true but only in terms of some of the matrices in the defining repetitive process state space model. Also the fact that no previous work has been done in this area and that these processes do have certain structural similarities with $1 \mathrm{D}$ discrete linear systems, makes it not an unreasonable place to start work. The most important conclusion to be drawn is, we argue, that it is indeed possible to control these processes in the presence of uncertainty in the defining model structure and that the results so obtained provide a useful benchmark for further work. Note also that alternative LMI settings for the subject area of this paper also exist - certainly in the case of 2D linear systems described by the Roesser model [16] and these should also be investigated in the repetitive process setting. Also the numerics associated with the resulting conditions may not always be well behaved and this area is again one which merits attention. 
The fact that the LMI approach is based on sufficient but not necessary conditions for stability along the pass raises the immediate question of whether an alternative starting point would yield better results in at least some cases. In which context, note that the stability test of Theorem 1 here can be formulated as a $\mu$ problem with two repeated complex blocks. To see this, first rewrite the condition of (3) as

$$
\operatorname{det}\left[I_{n+m}-\left[\begin{array}{cc}
z_{1} I_{n} & 0 \\
0 & z_{2} I_{m}
\end{array}\right] \Phi\right]=0
$$

or, equivalently,

$$
\operatorname{det}\left[I_{n+m}-\Phi^{\mathrm{T}}\left[\begin{array}{cc}
z_{1} I_{n} & 0 \\
0 & z_{2} I_{m}
\end{array}\right]\right]=0
$$

i.e.

$$
\operatorname{det}\left[I-\Phi^{\mathrm{T}} \Delta\right]=0
$$

with

$$
\Delta=\operatorname{diag}\left(z_{1} I_{n}, z_{2} I_{m}\right)
$$

which is similar to the $\mu$ problem with two repeated complex blocks see, for example, Reference [17] and for computational algorithms based on LMIs [18]. This general approach clearly has potential and requires an extensive investigation. Here this is left as a subject for further work with the note that the results given in this paper will provide a comparative benchmark for any algorithms arising from this $\mu$ analysis approach.

\section{REFERENCES}

1. Smyth KJ. Computer aided analysis for linear repetitive processes. PhD Dissertation, Department of Mechanical Engineering, University of Strathclyde, 1992.

2. Amann N, Owens DH, Rogers E. Predictive optimal iterative learning control. International Journal of Control 1998; 69(2):203-226.

3. Roberts PD. Stability analysis of iterative optimal control algorithms modeled as linear repetitive processes. Proceedings of The Institution of Electrical Engineers Part D 2000; 147(3):229-238.

4. Roesser RP. A discrete state space model for linear image processing. IEEE Transactions on Automatic Control 1975; AC-20(1):1-10.

5. Fornasini E, Marchesini G. Doubly-indexed dynamical systems: state space models and structural properties. Mathematical Systems Theory 1978; 12:59-72.

6. Rogers E, Owens DH. Stability Analysis for Linear Repetitive Processes, 1992, Lecture Notes in Control and Information Sciences Series, Vol 175. Springer Verlag: Berlin.

7. Rogers E, Lam J, Galkowski K, Xu S, Wood, J, Owens DH. LMI based stability analysis for discrete linear repetitive processes. Proceedings of 40th IEEE Conference on Decision and Control 2001; 4457-4462.

8. Galkowski K, Rogers E, Xu S, Lam J, Owens DH. LMIs-A fundamental tool in analysis and controller design for discrete linear repetitive processes. IEEE Transactions on Circuits and Systemas I: Fundamental Theory and Application 2002; 49(6):768-778.

9. Boyd S, Ghaoui LE, Feron E, Balakrishnan V. Linear Matrix Inequalities in Systems and Control Theory. SIAM Studies in Applied Mathematics. SIAM: Philadelphia, 1994.

10. Benton SE. Analysis and Control of Linear Repetitive Processes. PhD Thesis, University of Southampton, UK, 2000.

11. Bernussou J, Geromel JC, Peres, PLD. A linear programming oriented procedure for quadratic stabilization of uncertain systems. Systems and Control Letters 1989; 13:65-72.

12. de Oliveira M, Bernussou J, Geromel JC. A new discrete-time robust stability condition. Systems and Control Letters 1999; 37:261-265. 
13. Peaucelle D, Arzelier D, Bachelier O, Bernussou J. A new robust D-stability condition for polytopic uncertainty. Systems and Control Letters 2000; 40:21-30.

14. Anderson BDO, Agathoklis P, Jury EI, Mansour M. Stability and the matrix Lyapunov equation for discrete 2dimensional systems. IEEE Transactions on Circuits and Systems 1986; CAS-33:261-266.

15. Rogers E, Owens DH. Lyapunov stability theory and performance bounds for a class of $2 \mathrm{D}$ linear systems. Multidimensional Systems and Signal Processing 1996; 7:179-194.

16. Henrion D, Sdebek M, Bachelier O. Rank-one LMI approach to stability of 2-D polynomial matrices. Multidimensional Systems and Signal Processing 2001; 12:33-48.

17. Packard A, Doyle JC. The complex structured value. Automatica 1993; 29(1):71-109.

18. El Ghaoui L, Niculescu SI (Eds). Advances in Linear Matrix Inequality Methods in Control. SIAM, Philadelphia, 2000. 